\title{
Periodic boundary conditions for demagnetization interactions in micromagnetic simulations
}

\author{
K M Lebecki' ${ }^{1}$ M J Donahue ${ }^{2}$ and M W Gutowski ${ }^{1}$ \\ ${ }^{1}$ Institute of Physics, Polish Academy of Sciences, 02-668 Warsaw, Poland \\ ${ }^{2}$ National Institute of Standards and Technology, Gaithersburg, MD 20899-8910, USA
}

\begin{abstract}
A new method for the introduction of periodic boundary conditions to the self-magnetostatic (demagnetization) term in micromagnetic simulations is described, using an Ewald-like summation method in real space. The long-range character of the dipolar interactions is included without any distance cut-offs. The accumulated errors are carefully monitored to provide easy control of the quality of the results. This allows the calculations to be either accurate up to floating point limitations or less precise when computational speed requirements dominate. This method is incorporated into a full micromagnetic program, and comparisons are made to analytic results.
\end{abstract}

\section{Introduction}

Periodic boundary conditions (PBCs), also called Born-von Karman conditions [1], are a useful concept in many areas of physics. In micromagnetic simulations they allow efficient modelling of structures with certain geometries. In some sense, open boundary conditions include (or introduce) the effects of the sample surface while periodic (in space) boundary conditions neglect the influence of the sample surface in the analysed problem. One example where PBC may be successfully employed is in simulating arrays of periodically repeated structures, such as dots. This makes PBC an important tool for modelling and designing magnetic data storage devices. In principle, $\mathrm{PBC}$ s are applicable to infinite samples possessing a known periodicity. Often, however, finite samples of interest exceed by orders of magnitude the size limits that are computationally feasible using micromagnetic modelling. In such a case, one frequent approach is to model only a small part of the sample, for instance a small part of a computer hard disk structure containing a few ferromagnetic islands. If the simulation is simply truncated at the edges, the elongated nature of the sample is lost. PBCs are an important alternative for the investigation of such structures.

Among interactions that are usually considered in micromagnetism [2], exchange and demagnetization (dipole-dipole) are the ones that are non-local, and therefore require special implementation to handle PBC. The exchange interaction has, however, a short-range character making PBC implementation rather simple. In contrast, demagnetization interactions ('which originate from the classical interactions among the dipoles' [3]) have a long-range character, and implementation of PBC for this term is not trivial. A number of papers beginning in late 1980 s have suggested various solutions to this problem. One can simply neglect the presence of these interactions for distances exceeding a given critical value [4] or employ the idea of the Lorentz cavity [5]. Berkov has calculated the lattice sum using a modified Ewald method in the Fourier space [6]. As pointed out by Cohen [7] and developed by Mansuripur [8], in Fourier space one can analytically compute the dipole field arising from a sinusoidal magnetization pattern. This allows a straightforward computation of the dipole field using Fourier transforms. Programs based on fast multipole methods may use the idea of Apalkov and Visscher [9].

Despite such a wide spectrum of PBC implementation algorithms, PBCs are often simulated using the most simplified way: the magnetostatic interaction range is truncated to a given radius, as first proposed in [4]. Moreover, this cut-off is related not to an analysed and expected error, but rather to a fixed number of images (periodically repeated 'samples'). In this paper we reconsider the Ewald-like summation method, done 
in the real space. For simplicity, we focus our explanation on three-dimensional simulations with PBCs in one dimension (1D; the other two dimensions are given open boundary conditions). We also restrict to cubic discretization meshes. We show that introduction of far-field interaction cut-off can be avoided and that the choice of critical parameters may be done in a systematic way. Exploring the possible errors we show further that appropriate selection of parameters allows one to reduce the error to an arbitrary specified level, up to the limits imposed by floating point roundoff. We have implemented our algorithm [10] in the publicly available micromagnetic simulations package OOMMF [11]. Our simulation results are compared with theory for the infinite rod [12] and infinite prism.

The extension of our algorithm to non-cubic rectangular prism cells and two-dimensional PBCs is straightforward, but is not detailed in this paper. However, it should be pointed out that unlike boundary conditions that are periodic in one or two of the spatial dimensions, and despite suggestions to the contrary in some of the literature, boundary conditions that are periodic in three dimensions are problematic. We do not consider this case closely, except to point out some of the issues involved in section 3 .

\section{Principles of PBC}

In the numerical approach to micromagnetism the sample space is discretized and the sample is divided into cells. For a regular mesh of right rectangular prisms the cells can be indexed by triples $(i, j, k)$, where $i=1, \ldots, n_{x}$, $j=1, \ldots, n_{y}, k=1, \ldots, n_{z} ; n_{x} n_{y} n_{z}$ is the total number of cells. The inter-cell demagnetization interaction (intra-cell interaction is considered as well) can be evaluated using a generalized demagnetization tensor, $N$. In this paper we have used the tensor formulae given by Newell et al in [13]; similar formulations can be found in [14]. This tensor in Newell's formulation is obtained by calculating magnetic interactions assuming that every cell is uniformly magnetized and taking into account both the inter-cell offset and cell shape. (Another approach is also present in the literature, where for each cell the magnetization is not uniform but, for example, varies linearly in space [15].) In the non-PBC setting, the average effective demagnetization field at index $(i, j, k), \boldsymbol{H}_{\mathrm{d}}(i, j, k)$, can be expressed as

$\boldsymbol{H}_{\mathrm{d}}(i, j, k)=-\sum_{i^{\prime}, j^{\prime}, k^{\prime}=1}^{n_{x}, n_{y}, n_{z}} N\left(i-i^{\prime}, j-j^{\prime}, k-k^{\prime}\right) \boldsymbol{M}\left(i^{\prime}, j^{\prime}, k^{\prime}\right)$,

where $N(i, j, k)$ is the generalized demagnetization tensor for the interaction between cells; it depends on the relative position of the cells. The special case of self-demagnetization/intracell interaction, $N(0,0,0)$, is the common definition of the demagnetization tensor that can be found in many textbooks (e.g. [16]).

For ID PBC, we assume that a finite simulation window is repeated infinitely many times in one direction; see figure 1 . Each repetition will be called an image. In this paper we

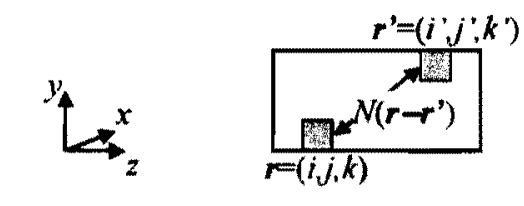

(a)

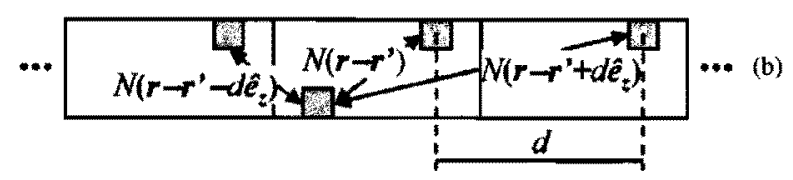

Figure 1. In the no-periodic boundary condition (no-PBC) case, $(a)$, the interaction between cells with indexes $(i, j, k)$ and $\left(i^{\prime}, j^{\prime}, k^{\prime}\right)$ is described by a demagnetization tensor, $N\left(i-i^{\prime}, j-j^{\prime}, k-k^{\prime}\right)$. With PBCs, (b), an infinite series of "images" has to be cvaluated. $N$ depends on the cells' relative position. $d$ is the period of the boundary conditions, $\hat{e}_{z}$ is the unit vector parallel to the $z$-axis. (Schematic picture; ID PBC are shown here.)

assume that the periodicity direction is parallel to the $z$-axis. The whole infinite sample can be enumerated with an infinite set of integers: $(i, j, k)$, where $i=1, \ldots, n_{x}, j=1, \ldots, n_{y}$, $k=-\infty, \ldots,+\infty$. The field in cell $(i, j, k)$ can be written as (see figure 1 ; image index $h=-1,0,1$ shown there)

$$
\begin{aligned}
& H_{\mathrm{d}}^{\mathrm{PBC}}(i, j, k) \\
& =-\sum_{i^{\prime}, j^{\prime}=1}^{n_{k}, n_{y}} \sum_{k^{\prime}=-\infty}^{+\infty} N\left(i-i^{\prime}, j-j^{\prime}, k-k^{\prime}\right) \boldsymbol{M}\left(i^{\prime}, j^{\prime}, k^{\prime}\right) \\
& =-\sum_{i^{\prime}, j^{\prime}=1}^{n_{x}, m_{y}} \sum_{h=-\infty}^{+\infty} \sum_{m^{\prime}=1}^{n_{z}} N\left(i-i^{\prime}, j-j^{\prime}, k-\left(h n_{z}+m^{\prime}\right)\right) \\
& \times \boldsymbol{M}\left(i^{\prime}, j^{\prime}, h n_{z}+m^{\prime}\right) \\
& =-\sum_{i^{\prime}, j^{\prime}=1}^{n_{x}, n_{y}} \sum_{h=-\infty}^{+\infty} \sum_{m^{\prime}=1}^{n_{z}} N\left(i-i^{\prime}, j-j^{\prime}, k-\left(h n_{z}+m^{\prime}\right)\right) \\
& \times \boldsymbol{M}\left(i^{\prime}, j^{\prime}, m^{\prime}\right) \\
& =-\sum_{i^{\prime}, j^{\prime}=1}^{n_{x}, n_{y}} \sum_{m^{\prime}=1}^{n_{z}}\left(\sum_{h=-\infty}^{+\infty} N\left(i-i^{\prime}, j-j^{\prime}, k-m^{\prime}+h n_{z}\right)\right) \\
& \times \boldsymbol{M}\left(i^{\prime}, j^{\prime}, m^{\prime}\right),
\end{aligned}
$$

where we introduce instead of $k^{\prime}$ two separate indexes: $h$ for enumeration of images and $m^{\prime}$ for the cells inside one image. We then exploit the periodicity of the magnetization (with period $n_{z}$ ), and at the end rearrange the summation and add parentheses to stress that the infinite summation concerns only the tensor. The interchange of the summations over $h$ and $m^{\prime}$ can be justified using the following argument: let $d=\left|\boldsymbol{r}(i, j, k)-\boldsymbol{r}\left(i, j, k+n_{z}\right)\right|$ be the period width (figure 1). Then, $N\left(i, j, k+h n_{z}\right)$ can be majorized by the dipole interaction of magnitude $O\left(|d h|^{-3}\right)$. Thus, the sums in (2) are absolutely convergent and Fubini's theorem can be applied. (We note in passing that this argument extends to the 2D PBC case as well, but in the 3D PBC setting the sum over $i, j, k$ is not absolutely convergent.) As a result, we arrive at a simple 
equation:

$$
\begin{aligned}
& \boldsymbol{H}_{\mathrm{d}}^{\mathrm{PBC}}(i, j, k)=-\sum_{i^{\prime}, j^{\prime}, k^{\prime}=1}^{n_{x}, n_{y}, n_{z}} N^{\mathrm{PBC}}\left(i-i^{\prime}, j-j^{\prime}, k-k^{\prime}\right) \\
& \quad \times \boldsymbol{M}\left(i^{\prime}, j^{\prime}, k^{\prime}\right),
\end{aligned}
$$

meaning that in the case of PBC the computation of the demagnetization interaction can be performed in the same way as without the PBC (see equation (1)), only the demagnetization tensor must be adapted to the $\mathrm{PBC}$ according to the following equation:

$$
N^{\mathrm{PBC}}(i, j, k)=\sum_{h=-\infty}^{+\infty} N\left(i, j, k+h n_{z}\right)
$$

Summarizing, introduction of $\mathrm{PBC}$ into a micromagnetic simulation package can be done by replacing the module where the demagnetization tensor is computed with one that evaluates (4). The tensor evaluation is typically done once at the start of the program, thus introduction of PBC need only affect the initialization stage.

\section{Problems with three periodic dimensions}

As noted in the introduction, one typically uses $\mathrm{PBCs}$ when one wants to ignore the effects of distant sample edges. This works if the periodic extension is one or two dimensional, because in that case the strength of the demagnetizing field arising from the sample edges decays to zero in the interior as the sample edges are made more distant. However, this is not the case in three dimensions. For example, consider a simulation window in the centre of a uniformly magnetized sphere. No matter how large the sphere is, the demagnetizing field throughout the sphere, and in particular inside the simulation window, is $-M / 3$. So, clearly, the field from the boundary of a threedimensional sample cannot be ignored. Nor is it easy to account for. As a second example, consider again a uniformly magnetized part, but suppose the outlying part shape is a prolate ellipsoid instead of a sphere. In this case the demagnetizing field is again non-zero, but it is not $-M / 3$ either. Indeed, the magnitude of the demagnetizing field will depend on the direction of the magnetization, being less than $M_{\mathrm{s}} / 3$ if the magnetization is aligned with the long axis of the ellipsoid, and greater than $M_{\mathrm{s}} / 3$ if the magnetization is in the plane orthogonal to the long axis.

The root of the problem is that the field from a single point dipole decays with distance as $1 /|r|^{3}$, and the sum across three dimensions, $\sum_{i} 1 /\left|\boldsymbol{r}_{i}\right|^{3}$, is divergent; in other words, the sum of the fields is not 'absolutely convergent.' Although there is cancellation between field terms from cells on various sides of the centre, so that the sum of the fields can be made convergent, the results obtained depend strongly on the manner in which the summation limit is taken to infinity, as seen from the sphere and ellipsoid examples. This is very different from the one- or two-dimensional PBC setting, which model physical systems of long needles or large flat plates, because magnetization aligned along the direction(s) of periodicity in those cases yields an effectively zero demagnetizing field. One may note that the dipole field terms arise from the average magnetization in the sample, and that the fields from higher order poles (quadrupoles, etc) are absolutely convergent even in three dimensions. So, in principle, it appears that well-defined threedimensional PBCs could be obtained by handling the field from the average magnetization component separately from the fields from higher order poles. Unfortunately, it is not clear how to compute the average magnetization field component if one allows for closure domains outside the simulation window at the boundaries of the physical system. Regardless, details of this approach are beyond the scope of this paper.

\section{Properties of the tensor $N^{\mathrm{PBC}}$}

The following statements are valid for regular meshes of rectangular cells:

(A) The demagnetization tensor for non-PBC conditions, $N(i, j, k)$, depends only on the relative position of the evaluated cells. This is a very important property (translation invariance) that allows the use of the convolution theorem and fast Fourier transform (FFT) algorithm in finite-difference programs ${ }^{3}$. The tensor $N^{\mathrm{PBC}}$ possesses the same feature.

(B) Another useful property of the demagnetization tensor concerns symmetries of its elements, $N_{x x}, N_{x y}, \ldots, N_{z z}$, which are all either even or odd in the $x-y$ - and $z$-coordinates [13]. We explain further this property in appendix $\mathrm{A}$, where we show that the same symmetries are valid for $\mathrm{PBC}$ tensor elements.

(C) The tensor defined in (4) is periodic in its third argument, with period $n_{z}$. The same applies, obviously, to the magnetization. This allows one to employ the convolution theorem in its native form, because both the 'signal' and 'response' functions have the same periodicity (see, for example [17]). The PBC calculations can thus be accelerated by avoiding zero padding in the periodic direction, a process that is unavoidable in non-PBC simulations ${ }^{4}$.

(D) Both $N$ and $N^{\mathrm{PBC}}$ are symmetric.

To summarize, the $\mathrm{PBC}$ tensor, $N^{\mathrm{PBC}}$, possesses the same important properties as the non-PBC tensor. Moreover, due to its periodicity, there is an additional possibility to accelerate simulations.

\section{Calculating infinite sum of tensors}

Evaluation of the sum in (4) must be done with care. First, an infinite number of summation elements must be handled via a finite number of numerical computations. Second, accumulation of numerical error must be considered. The first problem can be solved by the fact that the demagnetization interaction for large inter-cell distances can be approximated

3 In finite difference programs all cells have equal shape and size, thus the discretization mesh is regular.

4 There are, however, cases when the zero padding procedure may still be necessary; for example, because some FFT algorithms require the number of data channels (here: cells) to be a power of two. 


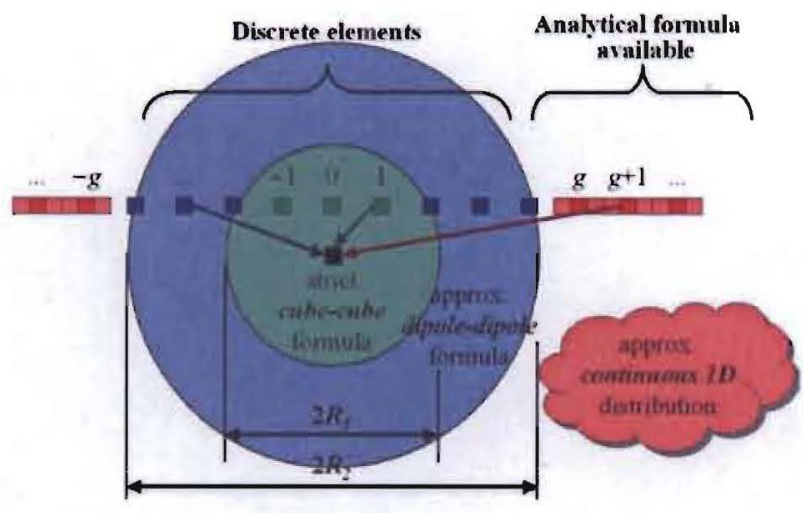

Figure 2. Schematic view: three methods used to compute the demagnetization tensor. Each method is applied in its own region. The square elements represent an individual cell and its images across multiple periods. Domain's boundary defined by the critical distance $R_{2}$ can be equivalently described by the $g$ index. (Colour online.)

by a continuous $I D$ magnetic charge/dipole distribution. For this approximation, discrete summation is replaced by integration, which is evaluated analytically. Thus, a procedure similar to the Ewald construction is used [18]: far cells are treated/computed differently than near ones; see figure 2 . We estimate the error introduced by the continuous $I D$ approximation. See appendix B for details. The critical distance, $R_{2}$, beyond which the continuous $I D$ calculation method can be used will be discussed shortly.

The second problem, i.e. error accumulated during the summation of elements with inter-cell distance smaller then $R_{2}$ (figure 2 ), requires a more complicated approach. First, we note that the formulae used to caleulate the demagnetization tensor suffer from numerical errors, which increase for larger inter-cell distances-see figure 3, grey points (red in online version). In figure 3 the errors in the calculated non-PBC tensor element, $\delta N_{x x}$, are shown as a function of the intercell distance. Let us mark $N_{\alpha \beta}$ as the $\alpha \beta$-element of the demagnetization tensor $(\alpha \beta=x x, x y, \ldots, z z)$. The error, $\delta N_{\alpha \beta}$, is defined as the difference between the exact tensor value calculated using arbitrary-precision methods, $N_{\alpha \beta}^{\mathrm{ex}}$, and the value returned by our ( 8 byte, double precision) floating point computations, $N_{\alpha \beta}^{\text {simul }}: \delta N_{\alpha \beta}=\left|N_{\alpha \beta}^{\text {simul }}-N_{\alpha \beta}^{\text {ex }}\right|$. See appendix B for details. Formulae used to compute the demagnetization tensor are quite simple and compact due to their recursive structure [13]. Yet, after resolving the recurrence complicated calculations exhibiting catastrophic eancellation appear [19]. This is the main source of the $\delta N$ errors. This method of computation will be called cube-cube below, as we have performed the analysis of the introduced errors for the case of cubic cells, even though the results also hold in general for other geometries. To deal with the errors causcd by the cube-cube method, we note that starting from a certain inter-cell distance, $R_{1}$, the demagnetization interaction can be successfully described neglecting the cell's geometry and approximating the cells by point dipoles ${ }^{5}$ - see

5 The formulae for point dipoles are present in tensor form in Newell's paper [13].

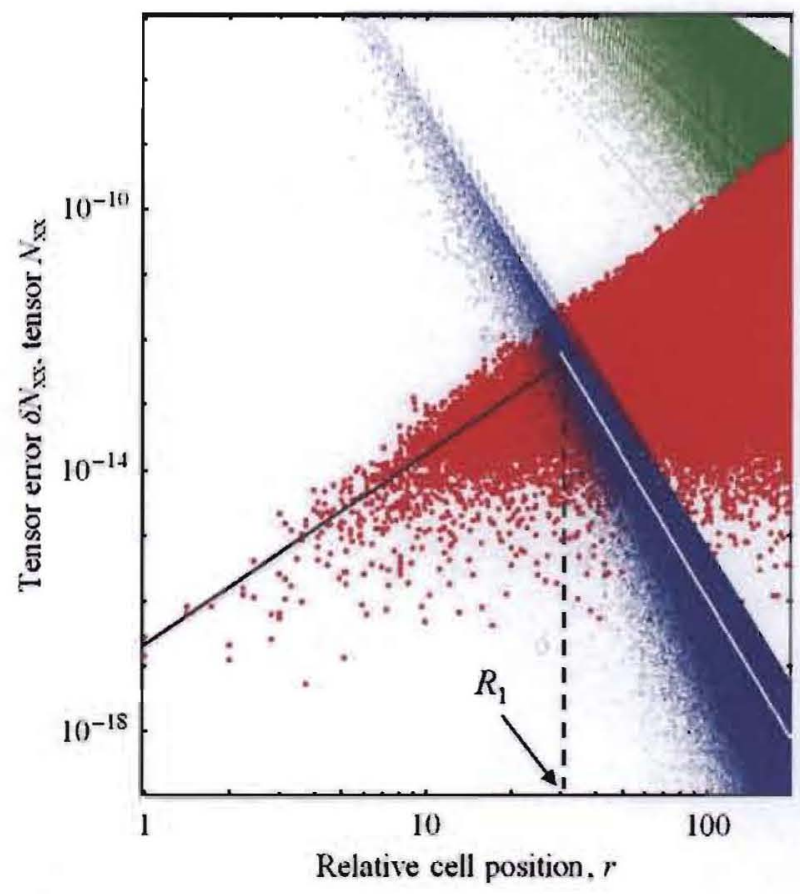

Figure 3. Errors in computation of non-PBC diagonal tensor elements, $\delta N_{x x}$, versus the relative cell position $r=|\boldsymbol{r}|$. Horizontal axis is in units of cubic cell edges. Grey points (red in online version): errors for formulas taking into account the cell's geometry (cube-cube method) [13], dark-grey/blue points: errors in case of point dipole approximation. Black and white lines are fits to data. For distance $r \approx 200$ the error of cube-cube method may already dominate the actual tensor value, $N_{x x}$ (light-grey/green points). See appendix B for details. (Colour online.)

figure 3 (dark-grey/blue points). The dipoles are placed at the centres of the cells and the value of each dipole moment is taken as appropriate for the cell's volume and magnetization. This tactic has one additional advantage: the dipolar formulae are much faster to compute than the full formulae of [13]. This computational method is henceforth called dipole-dipole. Again, this procedure is similar to the Ewald construction, delimiting now three spatial regions where we use different calculation approaches: the cube-cube method that takes into account the cell's geometry, the dipole-dipole method that approximates using point dipoles, and the continuous $1 D$ method that assumes continuous magnetization distributionsee figure 2. Deeper analysis of cube-cube errors together with a method to improve such calculations similar to our approach can be found in [19].

The errors in the PBC demagnetization tensor, computed using the approach described above, thus consist of three components: (i) numerical error caused by the cube-cube computation; (ii) method error resulting from the dipoledipole approximation and (iii) method error caused by the continuous $I D$ approximation. (There are additionally rounding errors arising from the summation of the cube-cube and dipole-dipole terms in (4), but these are comparatively minor.) The critical distance $R_{1}$ should be chosen to balance the errors caused by the cube-cube and dipole-dipole methods, according to figure 3 . The critical distance $R_{2}$ should be chosen to control the error introduced by the continuous $1 D$ 
method, $\delta N_{\alpha \beta}^{\mathrm{cont}}=\left|N_{\alpha \phi}^{\mathrm{con} t}-\sum N_{\alpha \beta}^{\mathrm{ex}}\right|$. (See appendix B for a computable estimate to $\delta N_{\alpha \beta}^{\text {cont }}$.) For this work $R_{2}$ was automatically selected so that $\delta N_{\alpha \beta}^{\text {cont }} / N_{\alpha \beta}<\varepsilon$, where $\varepsilon$ is the specified fidelity level ${ }^{6}$. Thus, it is possible to perform quicker and less accurate computations, by setting a higher value of $\varepsilon$ (which implies smaller $R_{2}$ ). It is however also possible to push the computations close to the precision limited by the available hardware. For example, for 8 byte floating point computations there are roughly 16 significant (decimal) digits, meaning minimal reasonable fidelity parameter value $\varepsilon \approx 10^{-16}$. Working with smaller $\varepsilon$ should result in no further quality improvement of the results. The algorithm we propose leads to a dynamic choice of parameter $R_{2}$, depending on the actual tensor element values, while $R_{1}$ is constant in our model.

Details of our error analysis are given in appendix $B$.

\section{Comparison with analytical theory}

We have tested the method described above by running our code [10] and simulating the hysteresis of an infinite cylindrical rod (with no magnetocrystalline anisotropy). We have performed the simulations using the following material parameters: exchange constant $A=9.604 \mathrm{pJ} \mathrm{m}^{-1}$ and saturation magnetization $M_{\mathrm{s}}=490 \mathrm{kA} \mathrm{m}^{-1}$. Thus, the exchange length was $\lambda_{\mathrm{ex}}=\sqrt{2 A /\left(\mu_{0} M_{\mathrm{s}}^{2}\right)} \approx 8 \mathrm{~nm}$. To avoid artificial symmetries we applied an offset field with varying randomly cell-to-cell direction and a constant value $\mu_{0}\left|\boldsymbol{H}_{\text {offset }}\right|=0.1 \mathrm{mT}$-similar to the simulations presented in [20]. The mesh cells were cubic. For rod's radius roughly equal to one (normalized, see below) the cell size was $(2.5 \mathrm{~nm})^{3}$, in all other cases the cell size was $(1.25 \mathrm{~nm})^{3}$. The mesh discretization leads to surface roughness that is obvious if one imagines the circular cross section of the rod as compared with its 'step-like' representation by the cubic cell mesh. It appears that 'staircase' edge-errors are more important for rods with larger radius and we had to use a special approach in that case-we employed the correction method described in [21]. Using this method, during the initialization stage correction factors were calculated by considering finer mesh (cell subdivision $64 \times 64 \times 1$ ) interacting over the range of seven local cells. These correction parameters have similar values as in [21], where they are labelled $q$ and $p$, respectively. For calculation of correction parameters we used the fidelity parameter $\varepsilon$ equal to $10^{-15}$. The PBC were applied along the $z$-axis, the $z$-axis being the long axis of the rod. Most of our simulations were done for a simulation window with the $z$-dimension (denoted here as $z$-length) equal to one cell. Except for reversal by buckling, as discussed below, tests with a larger $z$-length showed no visible change in the modelled coercivity. The hysteresis loops were rectangular (in agreement with [12]), so the nucleation field $\left|H_{n}\right|$ coincides with the coercive field. More details regarding PBC simulations for infinite 1D structures can be found in [20].

Analytical theory predicts three possible reversal modes for an infinite rod: coherent, buckling and curling [12]. The

6 This method works only for non-zero tensor element value $\left(\delta N_{\alpha f^{\prime}}^{\text {cont }}\right.$ is always nonnegative, see (B.3)).

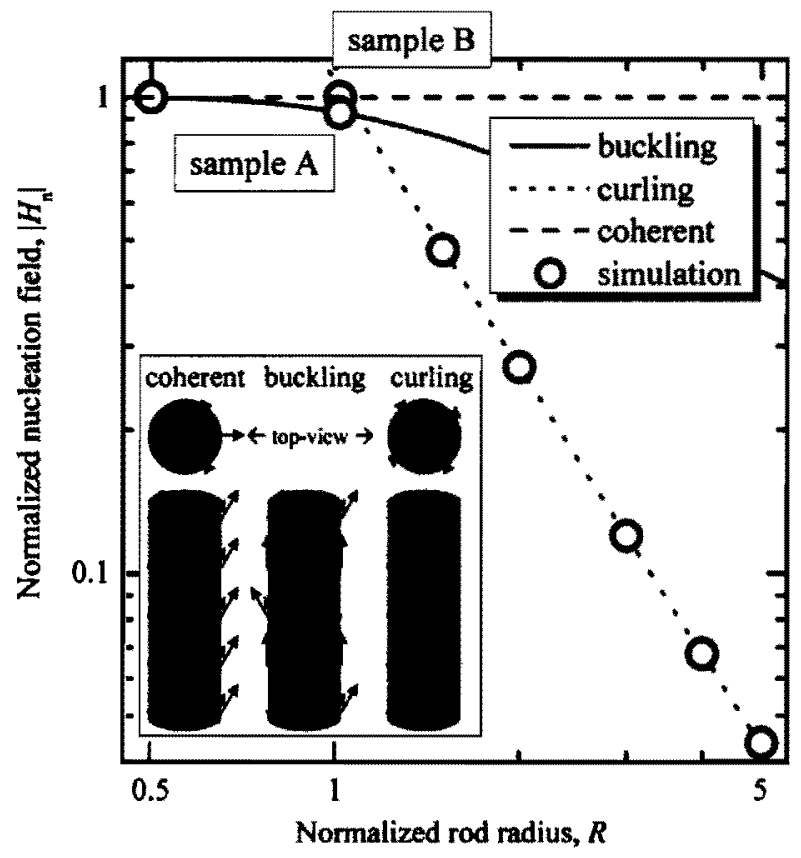

Figure 4. Comparison between theoretical predictions for the nucleation field of an infinite rod (lines) [12] and our simulations (open points). For radius $R \approx 1$, one simulation was done choosing the simulation window length equal to the buckling period (sample A). The other simulation was done assuming a one-cell length (sample B). The inset shows schematically the profiles of the three nucleation modes.

magnetization profile in the coherent and curling modes is independent of the $z$-position, while the buckling mode predicts a specific periodicity in the $z$-direction-see inset of figure 4. Dependence of the analytically computed nucleation field, $H_{\mathrm{n}}$, versus the rod's radius, $R$, for each mode is shown with lines in figure 4 . Both values $H_{\mathrm{n}}$ and $R$ are normalized similar to [12]. The field normalization factor is related to the demagnetization factor of the rod in the perpendicular direction ([3, chapter 9.2.1]); it is equal to $1 / 2 \mu_{0} M_{s} \approx 308 \mathrm{mT}$. The radius normalization factor is related to the exchange length; it is equal to $\sqrt{2 \pi} \lambda_{\text {ex }} \approx 20 \mathrm{~nm}$. Our simulation results (represented by points in figure 4) fit quite well the theoretical curves. Special attention is needed for the case $R \approx 1$. At this radius, buckling is the preferred reversal mode, so the model has to include the periodicity present in this mode. We have performed a series of tests for various sample $z$-lengths. Results are shown in figure 5 . For relatively small $z$-lengths the modelled coercivity matches the value predicted by the theory of coherent reversal, as in this range of $z$-lengths the expected buckling periodicity in the $z$-direction cannot be effectively rendered. For larger $z$-lengths the modelled results move closer to the expected value for the buckling theory. Best matching can be obtained when the $z$-length is a multiple of the expected period, but for larger $z$-length exact matching of the period length becomes less critical.

To check the errors introduced by our PBC approach we have also performed tests on infinite prisms with various rectangular cross sections, We assigned the prism walls 


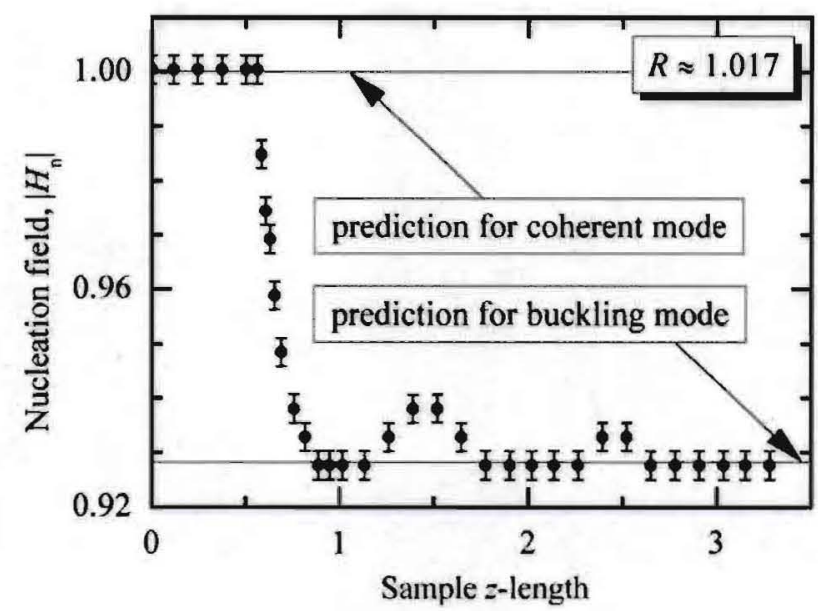

Figure 5. Nucleation field (vertical axis) modelled for different sample $z$-length (horizontal axis). The latter is normalized by the expected by theory [12] buckling period. Additional horizontal lines show predicted coercivity for the buckling and coherent mode. The error bars describe uncertainty due to steps in the applied magnetic field.

to be perpendicular to the $x$ - and $y$-axes. In this case there are no staircase edge-crrors. On the other hand, the demagnetization tensor in this case is complicated as it is not uniform inside the sample. It is possible, however, to define and analytically compute a volume-averaged demagnetization tensor $[13,22],\left\langle N^{\text {theory }}\right\rangle$. This volume-averaged tensor can be directly compared with an average of the tensor values across the cells of the sample as computed by our simulation, $\left\langle N^{\text {simul }}\right\rangle$. Regarding $\left\langle N^{\text {theory }}\right\rangle$, its off-diagonal elements are zero due to the fact that both the sample and the demagnetization tensor are symmetric. The diagonal element $\left\langle N^{\text {theory }}\right\rangle_{z z}$ is zero because of the infinite length of the prism along the $z$-direction. The two remaining elements, $\left\langle N^{\text {theory }}\right\rangle_{x x}$ and $\left\langle N^{\text {theory }}\right\rangle_{y y}$, can be found by evaluating the formulae from [13] in the limit of infinite $z$-edge, given the aspect ratio of the $x$-and $y$-edges ${ }^{7}$.

We performed simulations for structures having different dimensions $n_{x} \times n_{y} \times n_{z}: 2 \times 2 \times 2048,4 \times 4 \times 512,16 \times 16 \times 32$, $64 \times 64 \times 2,64 \times 64 \times 1,2 \times 512 \times 8,2 \times 256 \times 256$, $1 \times 128 \times 64,2 \times 64 \times 64,2 \times 16 \times 256,8 \times 16 \times 64$. (The computational cells are cubes, and $n_{x}, n_{y}, n_{z}$ denote the cell counts along each dimension.) The PBC were applied (as throughout this paper) along the $z$-axis. The errors, defined as $\delta\langle N\rangle=\left|\left\langle N^{\text {theory }}\right\rangle-\left\langle N^{\text {simul }}\right\rangle\right|$, are generally qualitatively similar for all considered cases. Major differences between these structures concern the $g$ values, see below. The largest errors, found in the structure $16 \times 16 \times 32$, are shown in figure 6 (solid symbols). We present here $\delta\langle N\rangle_{x x}$ and $\delta\langle N\rangle_{z z}$ elements plotted versus the fidelity level, $\varepsilon$, defined in the

\footnotetext{
7 We have used the Mathematica package for this purpose. We have checked different precision settings to make sure that rounding errors are not affecting the result. The trace of the tensor has to be equal to one, so actually it is enough to compute just one tensor element, e.g., $\left\langle N^{\text {theory }}\right\rangle_{x x}$. Note: commercial equipment and software referred to in this paper are identified for informational purposes only, and does not imply recommendation of or endorsement by the National Institute of Standards and Technology, nor does it imply that the products so identified are necessarily the best available for the purpose.
}

Summation elements, $g$

294

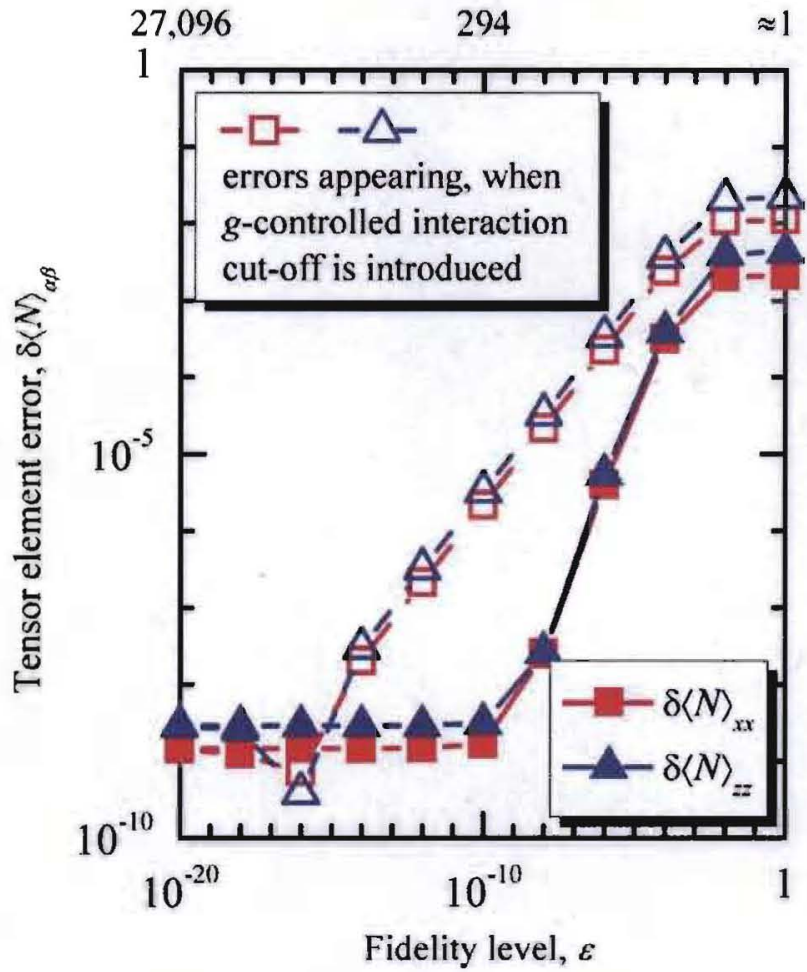

Figure 6. Errors in the computed average demagnetization tensor elements $\delta\langle N\rangle_{x x}$ (squares) and $\delta\langle N\rangle_{z z}$ (triangles) for an infinite rectangular prism as a function of the fidelity level, $\varepsilon$ (solid points) Simulation window size: $n_{x} \times n_{y} \times n_{z}=16 \times 16 \times 32$. The errors are reduced with smaller values of $\varepsilon$ until bottoming out at $\varepsilon \approx 10^{-12}$ to $10^{-10}$. Open points represent the situation when no continuous $1 D$ calculation is done, i.e. when a cut-off in the dipolar interaction is introduced. The upper horizontal axis gives the image index $g$ beyond which the continuous $I D$ calculation is used.

previous section ${ }^{8}$. The upper horizontal axis shows the image index $g$ beyond which the continuous $1 D$ calculation is used, i.e. roughly half the number of discrete elements indicated in figure 2. Certainly, a lower $\varepsilon$ parameter requires a higher value of $g$. However, exact $g$ values depend strongly on the simulation window geometry, mainly on its aspect ratio ${ }^{9}$. Therefore the fidelity level is a better parameter for setting the $R_{2}$ radius (figure 2 ) than a fixed value of $g$. In figure 6 , as expected, the errors get smaller for smaller values of $\varepsilon$. As we work with 16 digits precision, one might expect that using $\varepsilon$ smaller then $10^{-16}$ will have no further influence on the errors (see previous section). Actual error bottoms out at a somewhat larger value, primarily due to errors in the evaluation of the individual tensor elements, as illustrated in figure 3 .

\footnotetext{
8 The $\delta\langle N\rangle_{y y}$ error is comparable to $\delta\langle N\rangle_{x x}$. The off-diagonal $(\alpha \neq \beta)$ $\delta\langle N\rangle_{\alpha \beta}$ errors are much smaller. $\delta\langle N\rangle_{x y}$ is constant as a function of $\delta$ and equal roughly to $10^{-17} . \delta\langle N\rangle_{x z}$ is about $10^{-20}$ for $\varepsilon \approx 1$, for smaller $\varepsilon$ it falls down, at $\varepsilon \approx 10^{-12}$ it bottoms out with value ca $10^{-29}$. These values, being based on averages across the simulation window, are probably not representative of errors in the individual terms, but rather reflect error cancellations arising from problem symmetry.

9 Particularly, in the case of $z$-length equal to one cell, $g$ is exceptionally large.
} 
We have also tested the importance of the continuous $1 D$ calculations. Dipolar interactions have a long-range character, so it is crucial to consider their presence up to infinity. Open points in figure 6 reflect the case when there are no continuous $I D$ components in the calculation of the $N^{\mathrm{PBC}}$ tensor, i.e. a $g$-controlled cut-off is introduced in the interaction. One sees that already for $\varepsilon \approx 1$ introduction of the cut-off increases the error by one order of magnitude. For more accurate computations (smaller $\varepsilon$ ) this effect is even stronger, leading to errors three orders of magnitude larger for $\varepsilon=10^{-10}$.

\section{Summary and conclusions}

This paper describes a method of modification of the demagnetization tensor $N$ for incorporating ID PBCs into micromagnetic simulations. The magnetostatic effects from each cell and its infinitely repeated images are handled by dividing the images into three domains. In the near-field domain, the effects are computed using analytic formula for the interaction between two uniformly magnetized cells. Outside the near-field, the analytic formulae are numerically inaccurate, so in the mid-field domain the effects are computed using discrete dipole-dipole interactions, which are also computed more quickly than the analytic formula. In the extreme far-field, the discrete dipoles are replaced with a continuous dipole distribution, thereby alleviating the truncation error that would occur if the images were simply ignored beyond some point. The domain limits are determined in a systematic way to control error. Analysing the errors we conclude that they can be easily controlled by the fidelity parameter, $\varepsilon$, allowing accurate modelling for small values of $\varepsilon$. This control may be weakened if desired for faster but less precise computations. Either way, the PBC calculations affect only the determination of $N$ in the initialization stage of the simulation. As compared with a non-PBC simulation, the computational cost of the remainder of the simulation is the same, or even reduced in cases where zero padding can be eliminated. We also show that the results of our algorithm are consistent with analytic predictions. Although only the 1D PBC with cubic cells setting is considered here, the extensions to two dimensions and non-cubic (rectangular prism) cells are straightforward (though computationally more challenging).

Important aspects of our approach include full control of accumulated errors and the importance of including dipole interactions up to infinity. It is hoped that these considerations will be useful in other $\mathrm{PBC}$ algorithms as well, involving, for example, the magnetic scalar potential.

\section{Acknowledgments}

We acknowledge support from the Polish Ministry of Science and Higher Education (Grant No 1346/B/H03/2007/33) and from the European agency COST Action P19 (WG3).

\section{Appendix A. Symmetry rules for $N^{\mathrm{PBC}}$ tensor elements}

The demagnetization tensor elements possess certain symmetry properties regarding the reflection of coordinates. The diagonal elements are even: $N_{\alpha \alpha}(i, j, k)=N_{\alpha \alpha}(-i, j, k)=$ $N_{\alpha \alpha}(i,-j, k)=N_{\alpha \alpha}(i, j,-k)$, while the off-diagonal elements, $N_{\alpha \beta},(\alpha \neq \beta)$, are odd in $\alpha$ and $\beta$ and even in the third direction, e.g. $N_{x y}(i, j, k)=-N_{x y}(-i, j, k)=$ $-N_{x y}(i,-j, k)=N_{x y}(i, j,-k)$ [13]. To evaluate in this appendix the properties of the $N^{\mathrm{PBC}}$ tensor, we consider one tensor element, $N_{\alpha \beta}$ (diagonal or off-diagonal), that is either even $(\gamma=+1)$ or odd $(\gamma=-1)$ in the $z$-coordinate. Naming the operation of reflection of the $z$-coordinate $\sigma_{z}$, we thus have the relation $\sigma_{z}\left(N_{\alpha \beta}\right)=\gamma N_{\alpha \beta}$. The symmetry of the tensor element can then be written as

$\sigma_{z}\left(N_{\alpha \beta}(i, j, k)\right)=N_{\alpha \beta}(i, j,-k)=\gamma N_{\alpha \beta}(i, j, k)$.

Application of the $\sigma_{z}$ transformation to the ID PBC tensor element is shown below (PBCs are applied along the $z$-coordinate, $n_{z}$ is the number of cells in the periodicity direction).

$$
\begin{aligned}
& \sigma_{z}\left(N_{\alpha \beta}^{\mathrm{PBC}}(i, j, k)\right)=N_{\alpha \beta}^{\mathrm{PBC}}(i, j,-k) \\
& \quad=\sum_{h=-\infty}^{+\infty} N_{\alpha \beta}\left(i, j,-k+h n_{z}\right) \\
& \quad=\sum_{h=-\infty}^{+\infty} \gamma N_{\alpha \beta}\left(i, j, k-h n_{z}\right) \\
& \quad=\gamma \sum_{h^{\prime}=-\infty}^{+\infty} N_{\alpha \beta}\left(i, j, k+h^{\prime} n_{z}\right)=\gamma N_{\alpha \beta}^{\mathrm{PBC}}(i, j, k) .
\end{aligned}
$$

The case of reflection of the $x$ - or $y$-axis is even simpler; a few algebraic transformations show that the symmetry is retained also in this situation.

Thus, we have shown that $\mathrm{PBC}$ and non-PBC tensors have the same symmetries regarding the reflection of coordinates.

\section{Appendix B. Errors occurring while evaluating the tensor $N^{\mathrm{PBC}}$}

In our following analysis we focus on cubic cells, though the ideas presented in this appendix may be applied to right rectangular prisms as well. We mark $N_{\alpha \beta}(i, j, k)$ as the $\alpha \beta$-element of the tensor $(\alpha \beta=x x, x y, \ldots, z z)$ for interaction between cells with relative position vector $r=(i, j, k)$. Distance-related values are given in units of cell edges, thus, e.g. $N(1,0,0)$ denotes interaction between a pair of two adjacent cells having common $Y Z$ wall. Such an approach is possible due to the scaling properties of $N$ (for $N^{\mathrm{PBC}}$ they are valid as well): this tensor remains 'unchanged if all quantities are scaled by the same amount' [13]. The data presented here (except $N^{\mathrm{ex}}$, see below) are results of computations done in 'double' ( 8 byte) floating point arithmetic with roughly sixteen decimal digits precision ${ }^{10}$. First, we will

${ }^{10}$ Environment: gcc compiler v. 3.2, o/s Windows 2000, Intel 32-bit processor. 
describe in detail the errors in the cube-cube and dipoledipole methods, then we will discuss the continuous $I D$ error.

To evaluate the errors in the cube-cube and the dipoledipole calculation methods, we have considered a limited 3D space region, where the relative $i / j / k$ indices of the cells were in the range $0-200$. Due to certain symmetries valid for cubic cells, such as $N_{x x}(i, j, k)=N_{y y}(j, i, k)$, it is enough to evaluate diagonal tensor elements $N_{x x}$ and offdiagonal $N_{x y}$. Computational error of the cube-cube method, $\delta N^{c c}$, is defined as the absolute difference between a tensor element calculated using this method, $N^{c c}$, and a supposed exact value, $N^{\mathrm{ex}}: \delta N^{\mathrm{cc}}=\left|N^{\mathrm{cc}}-N^{\mathrm{ex}}\right|$. Error of the dipoledipole tensor value, $N^{d d}$, is defined in an analogous way: $\delta N^{\mathrm{dd}}=\left|N^{\mathrm{dd}}-N^{\mathrm{ex}}\right|$. We have obtained the exact values, $N^{\mathrm{ex}}$, using Newell's formulae [13] in high-precision calculations with the Mathematica package, where we used so-called 'arbitrary-precision numbers' with sixteen accurate digits. The internal intermediate calculations were performed using on-demand up to 50 digits precision (by setting Mathematica's variable $\$$ MaxExtraPrecision) ${ }^{11}$. In the case of diagonal tensor elements, values of $\delta N^{\mathrm{cc}}$ and $\delta N^{\mathrm{dd}}$ plotted against the intercell distance, $r=|r|$, are shown in figure 3 (grey/red and dark-grey/blue points, respectively). A picture drawn for offdiagonal elements looks very similar. Clearly, the cube-cube method works better for smaller distances, while the dipoledipole method introduces smaller errors for larger distances. For $r \approx 200$ the cube-cube errors are so significant that they may dominate the actual tensor value (light-grey/green points). Treating the errors in a stochastic way and fitting the data with a polynomial function, $\delta N(r)=A r^{p}$, we obtain the following fit parameters for diagonal elements:

$$
\begin{aligned}
& A_{x x}^{\mathrm{ce}} \approx 2.2 \times 10^{-17}, \quad p_{x x}^{\mathrm{ce}} \approx 2.9, \\
& A_{x x}^{\mathrm{dd}} \approx 0.023, \quad p_{x x}^{\mathrm{dd}} \approx-7.2,
\end{aligned}
$$

for cube-cube and dipole-dipole methods, respectively. Based on the intersection of these two functions, the critical distance can be estimated as $R_{1}\left(N_{x x}\right) \approx 31$. For off-diagonal tensor elements the parameters are

$A_{x y}^{\mathrm{cc}} \approx 1.2 \times 10^{-17}, \quad p_{x y}^{\mathrm{cc}} \approx 3.1$,

$A_{x y}^{\mathrm{dd}} \approx 0.011, \quad p_{x y}^{\mathrm{dd}} \approx-6.9$,

and again $R_{1}\left(N_{x y}\right) \approx 31$.

Concerning the error of the continuous $I D$ method, $\delta N^{\text {eont }}$, we have approximated it in following way:

$$
\begin{gathered}
\delta N^{\mathrm{cont}}=\left|N^{\mathrm{cont}}-\sum N^{\mathrm{ex}}\right| \leqslant\left|N^{\mathrm{cont}}-\sum N^{\mathrm{dd}}\right| \\
+\left|\sum N^{\mathrm{dd}}-\sum N^{\mathrm{ex}}\right|
\end{gathered}
$$

where $N^{\text {cont }}$ is the value of the tensor calculated in the continuous $1 D$ method (see (B.5)). We point out that $\delta N^{\text {cont }}$ and $N^{\mathrm{comt}}$ are defined via the sum over PBC images. The last

11 For diagonal tensor calculations we occasionally received a message stating that \$MaxExtraPrecision is too low to achieve desired 16 -digits precision. This affects the error estimation parameters (B.1) and (B.2) invisibly. term in the right-hand side of (B.3) can be estimated using (B.1) and (B.2): $\left|\sum N^{\mathrm{dd}}-\sum N^{\mathrm{ex}}\right| \leqslant \sum\left|N^{\mathrm{dd}}-N^{\mathrm{ex}}\right|=\sum \delta N^{\mathrm{dd}}$.

To evaluate the remaining term $\left|N^{\text {cont }}-\sum N^{\text {dd }}\right|$, we will first derive the formulae for $N^{\text {cont }}$. We mark the magnetization of a cell as $\boldsymbol{M}$, and the volume of the cell as $V$. In the point dipole approximation, the demagnetization field around the cell, at offset $\boldsymbol{r}=(i, j, k)$, is $\boldsymbol{H}_{\mathrm{d}}(\boldsymbol{r})=$ $\left(3 \boldsymbol{r}(V \boldsymbol{M} \cdot \boldsymbol{r})-V \boldsymbol{M}|\boldsymbol{r}|^{2}\right) /\left(4 \pi|\boldsymbol{r}|^{5}\right)$. The continuous $1 D$ method is used for distant cells, where $|\boldsymbol{r}|>R_{2}$ (figure 2). Thus, we are interested in the summation $\sum_{h=g}^{\infty} \boldsymbol{H}_{\mathrm{d}}\left(i, j, k+h n_{z}\right)+$ $\sum_{h=-\infty}^{-\infty} H_{\mathrm{d}}\left(i, j, k+h n_{z}\right)$, where the cut-off index $g\left(R_{2}\right)$ depends on the radius $R_{2}$ (see (4) and figure 2). We point out that as we use here units of cell edges, the length $d$ is equal to $n_{2}$, the volume $V$ is equal to one, and the $(0,0,0)$ cell is chosen in such a way that the $(i, j, k)$ indexes are nonnegative. We introduce a function

$f(z)=\frac{1}{4 \pi d} \frac{3\left(r+\hat{e}_{z} z\right)\left(M \cdot\left(r+\hat{e}_{z} z\right)\right)-M\left|r+\hat{e}_{z} z\right|^{2}}{\left|r+\hat{e}_{z} z\right|^{5}}$.

Now, the continuous $I D$ approximation is equivalent to the following approximation:

$$
\begin{aligned}
& \sum_{k=8}^{\infty} \boldsymbol{H}_{\mathrm{d}}(i, j, k+h d)+\sum_{h=-g}^{-\infty} \boldsymbol{H}_{\mathrm{d}}(i, j, k+h d) \\
& \quad \approx \int_{d(g-1 / 2)}^{\infty} f(z) \mathrm{d} z+\int_{-\infty}^{-d(g-1 / 2)} f(z) \mathrm{d} z \stackrel{\text { def }}{=}-N^{\mathrm{zont}} \boldsymbol{M} .
\end{aligned}
$$

The integral $\int f(z) \mathrm{d} z$ can be evaluated in a closed form. Thus, the elements of the tensor $N^{\text {cont }}$ can be derived (the case $i=j=0$ for $N_{x x}^{\text {cont }}$ and $N_{x y}^{\text {cont }}$ is handled separately; also as we are interested in comparatively large $R_{2}$ values, we assume that $g>k / d+1 / 2$, thus $g \geqslant 2$ )

$$
\begin{aligned}
& N_{x x}^{\mathrm{cont}}(i, j, k)=\frac{\left(j^{2}-i^{2}\right)}{4 \pi d\left(i^{2}+j^{2}\right)^{2}} \\
& \times\left(2+\frac{k+d(1 / 2-g)}{\left(i^{2}+j^{2}+(k+d(1 / 2-g))^{2}\right)^{3 / 2}}\right. \\
& \times\left(j^{2}+\frac{2 i^{4}}{i^{2}-j^{2}}+(k+d(1 / 2-g))^{2}\right) \\
& -\frac{k-d(1 / 2-g)}{\left(i^{2}+j^{2}+(k-d(1 / 2-g))^{2}\right)^{3 / 2}} \\
& \left.\times\left(j^{2}+\frac{2 i^{4}}{i^{2}-j^{2}}+(k-d(1 / 2-g))^{2}\right)\right) \\
& N_{x y}^{\text {cont }}(i, j, k)=\frac{-i j}{4 \pi d\left(i^{2}+j^{2}\right)} \\
& \times\left(\frac{4}{i^{2}+j^{2}}+\frac{k+d(1 / 2-g)}{\left(i^{2}+j^{2}+(k+d(1 / 2-g))^{2}\right)^{3 / 2}}\right. \\
& \times\left(3+\frac{2(k+d(1 / 2-g))^{2}}{i^{2}+j^{2}}\right) \\
& -\frac{k-d(1 / 2-g)}{\left(i^{2}+j^{2}+(k-d(1 / 2-g))^{2}\right)^{3 / 2}} \\
& \left.\times\left(3+\frac{2(k-d(1 / 2-g))^{2}}{i^{2}+j^{2}}\right)\right)
\end{aligned}
$$




$$
\begin{array}{r}
N_{x z}^{\mathrm{cont}}(i, j, k)=\frac{i}{4 \pi d} \\
\times\left(\left(i^{2}+j^{2}+(k+d(1 / 2-g))^{2}\right)^{-3 / 2}\right. \\
\left.-\left(i^{2}+j^{2}+(k-d(1 / 2-g))^{2}\right)^{-3 / 2}\right), \\
N_{z z}^{\mathrm{cont}}(i, j, k)=\frac{1}{4 \pi d} \\
\times\left(\frac{k+d(1 / 2-g)}{\left(i^{2}+j^{2}+(k+d(1 / 2-g))^{2}\right)^{3 / 2}}\right. \\
\left.-\frac{k-d(1 / 2-g)}{\left(i^{2}+j^{2}+(k-d(1 / 2-g))^{2}\right)^{3 / 2}}\right),
\end{array}
$$

$N_{y y}^{\text {cont }}(i, j, k)=N_{z z}^{\text {cont }}(j, i, k)$,

$N_{y z}^{\text {cont }}(i, j, k)=N_{x z}^{\text {cont }}(j, i, k)$.

$N_{x x}^{\text {cont }}$ and $N_{x y}^{\text {cont }}$ have the following form for the special case of $i=j=0$ :

$$
\begin{aligned}
& N_{x x}^{\text {cont }}(0,0, k)=\frac{1}{8 \pi d}\left((k+d(1 / 2-g))^{-2}\right. \\
& \left.\quad+(k-d(1 / 2-g))^{-2}\right), \\
& N_{x y}^{\text {cont }}(0,0, k)=0 .
\end{aligned}
$$

Knowing $N^{\text {cont }}$, the term $\left|N^{\text {cont }}-\sum N^{\mathrm{dd}}\right|$ from equation (B.3) can be evaluated. We do it using two power series expansions. The first time to approximate $N^{\text {dd }}$ to make possible calculating the sum $\sum N^{\mathrm{dd}}$. As this sum is known with a precision $O\left(g^{-\gamma}\right)$, we use a power series expansion to write the whole expression in a compatible fashion. Taking the $x y$ element as an example:

$$
\begin{aligned}
& N_{x y}^{\text {cont }}- \sum N_{x y}^{\mathrm{dd}}=N_{x y}^{\mathrm{cont}}(i, j, k) \\
&-\left(\sum_{h=g}^{\infty} N_{x y}^{\mathrm{dd}}(i, j, k+h d)+\sum_{h=-g}^{-\infty} N_{x y}^{\mathrm{dd}}(i, j, k+h d)\right) \\
&= N_{x y}^{\mathrm{cont}}(i, j, k) \\
&-\frac{i j}{\pi} \sum_{h=g}^{\infty}\left(\frac{3}{2 d^{5}} h^{-5}+\frac{15\left(i^{2}+j^{2}-6 k^{2}\right)}{4 d^{7}} h^{-7}+O\left(h^{-9}\right)\right) \\
&= \frac{-5 i j}{16 \pi d^{5}} g^{-6}+O\left(g^{-7}\right) . \\
&\left(N_{x y}^{\text {cont }}-\sum N_{x y}^{\mathrm{dd}}=0 \text { for } i=j=0 .\right)
\end{aligned}
$$

Following are the results for the remaining elements:

$$
\begin{aligned}
& N_{x x}^{\mathrm{cont}}-\sum N_{x x}^{\mathrm{dd}}=\frac{1}{16 \pi d^{3}} g^{-4}+O\left(g^{-5}\right), \\
& N_{x z}^{\mathrm{cont}}-\sum N_{x z}^{\mathrm{dd}}=\frac{5 i k}{4 \pi d^{5}} g^{-6}+O\left(g^{-7}\right), \\
& N_{z z}^{\mathrm{cont}}-\sum N_{z z}^{\mathrm{dd}}=\frac{-1}{8 \pi d^{3}} g^{-4}+O\left(g^{-5}\right), \\
& N_{y y}^{\mathrm{cont}}-\sum N_{y y}^{\mathrm{dd}}=\frac{1}{16 \pi d^{3}} g^{-4}+O\left(g^{-5}\right), \\
& N_{y z}^{\mathrm{cont}}-\sum N_{\mathrm{yz}}^{\mathrm{dd}}=\frac{5 j k}{4 \pi d^{5}} g^{-6}+O\left(g^{-7}\right)
\end{aligned}
$$

Our approach might be developed further, e.g. by estimating the total error of the computed tensor element and/or checking the trace of the demagnetization tensor, which should be equal to zero for non-overlapping interacting cells [13]. Generally speaking, the whole tactic presented in figure 2 is based on power series expansion, both in construction of $N^{\mathrm{dd}}$ and $N^{\text {cont }}$. Thus, improvements may be had by including higher order elements, leading to a decrease in computational effort and final error. This might be especially useful in the case of 2D PBC or non-cubic cells.

\section{References}

[1] Born M 1923 Atomtheorie des festen Zustandes (Dynamik der Kristallgitter) (The International Series of Monographs on Physics vol 25) (Leipzig/Berlin: G.B. Teubner) pp 587-8

[2] Brown W F Jr 1963 Micromagnetics (Interscience Tracts on Physics and Astronomy vol 18) ed R E Marshak (New York: Wiley)

[3] Aharoni A 2000 Introduction to the Theory of Ferromagnetism (International Series of Monographs on Physics vol 109) ed J Birman and S F Edwards (New York: Oxford University Press)

[4] Zhu J-G and Bertram H N 1988 Micromagnetic studies of thin metallic films J. Appl. Phys. 63 3248-53

[5] Vos M J, Brott R L, Zhu J G and Carlson L W 1993 Computed hysteresis behavior and interaction effects in spheroidal particle assemblies IEEE Trans. Magn. 29 3652-7

[6] Berkov D V and Gorn N L 1998 Quasistatic remagnetization processes in two-dimensional systems with random on-site anisotropy and dipolar interaction: numerical simulations Phys. Rev. B 57 14332-43

[7] Cohen $M H$ and Keffer $F 1955$ Dipolar sums in the primitive cubic lattices Phys. Rev. 991128

[8] Mansuripur M and Giles R 1988 Demagnetizing field computation for dynamic simulation of the magnetization reversal process IEEE Trans. Magn. 242326

[9] Apalkov D M and Visscher P B 2003 Fast multipole method for micromagnetic simulation of periodic systems IEEE Trans. Magn. 39 3478-80

[10] Lebecki K M PBC Module for OOMMF. See: http://info.ifpan.edu.pl/ $\sim$ lebecki/pbc

[11] Donahue M J and Porter D G 1999 OOMMF User's Guide, release 1.2a4pre Report NISTIR 6376 (Gaithersburg, MD: NIST). See: http://math.nist.gov/oommf

[12] Aharoni A and Shtrikman S 1958 Magnetization curve of the infinite cylinder Phys. Rev. 1091522

Frei E H, Shtrikman S and Treves D 1957 Critical size and nucleation field of ideal ferromagnetic particles Phys. Rev. 106446

[13] Newell A J, Williams W and Dunlop D J 1993 A generalization of the demagnetizing tensor for nonuniform magnetization J. Geophys. Res. [Atmos.] 98 9551-5

[14] Fukushima H, Nakatani $Y$ and Hayashi N 1998 Volume average demagnetizing tensor of rectangular prisms $I E E E$ Trans. Magn. 34 193-8

Schabes M E and Aharoni A 1987 Magnetostatic interaction fiejds for a three-dimensional array of ferromagnetic cubes IEEE Trans. Magn. MAG-23 3882-8

[15] Labrune $M$ and Miltat J 1995 Wall structures in ferro/antiferromagnetic exchange-coupled bilayers: a numerical micromagnetic approach J. Magn. Magn. Mater. $151231-45$

Ramstock K, Leibl T and Hubert A 1994 Optimizing stray field computations in finite-element micromagnetics J. Magn. Magn. Mater. $13597-110$ 
[16] Blundell S 2003 Magnetism in Condensed Matter (Oxford Master Series in Condensed Matter Physics) (New York: Oxford University Press)

[17] Brigham E O 1988 The Fast Fourier Transform and lts Applications (Prentice Hall Signal Processing Series) (Englewood Cliffs, NJ: Prentice-Hall) pp 118-30

[18] Ewald P P 1913 Zur Theorie der Interferenzen der Röntgenstrahlen in Kristallen Phys. Z. 14 465-72

[19] Donahue M J Accurate computation of the demagnetization tensor, unpublished
[20] Lebecki K M 2007 Magnetization reversal modeling for long ferromagnetic nanotubes Mater. Sci-Poland at press

[21] Donahue M J and McMichael R D 2007 Micromagnetics on curved geometries using rectangular cells: error correction and analysis IEEE Trans, Magn. 43 2878-80

[22] Brown W F Jr 1962 Magnetostatic Principles in Ferromagnetism (Selected Topics in Solid State Physics) (Amsterdam: North-Holland) 